\title{
Le baobab, un arbre emblématique dans le futur urbain du pôle de Diamniadio au Sénégal : marqueur spatial, représentation sociale et intégration paysagère
}

\author{
Sidia Diaouma Badiane ${ }^{a}$, Edmée Mbaye ${ }^{b}$
}

RÉSUMÉ. L'intégration de l'arbre dans la ville est devenue un enjeu de taille en matière de planification urbaine. Les projets de villes nouvelles tentent de prendre en compte cette dimension. La création du pôle urbain de Diamniadio au Sénégal s'inscrit ainsi dans cette logique d'intégration paysagère. Cet article s'intéresse à l'image du baobab dans la construction de ce pôle urbain. Notre hypothèse est que cet arbre bénéficie d'une attention particulière en raison de son statut d'espèce protégée (il constitue d'ailleurs un des deux sceaux symboliques du Sénégal), mais également du fait qu'il fournit à la population des biens et services écosystémiques remarquables. Cette étude s'appuie sur des données d'inventaire des baobabs dans le pôle urbain de Diamniadio et sur des données d'enquête (quantitatives et qualitatives). Le recensement de baobabs in situ a permis d'identifier 333 individus répartis de façon aléatoire dans l'espace. Malgré la croissance urbaine, le baobab garde toujours son aspect d'arbre symbolique, gravé dans la mémoire collective.

ABSTRACT. Integrating trees in a city has become very important in urban planning. Project personnel in new cities are attempting
to include trees in their planning. The creation of the Diamniadio urban centre in Senegal is an example of landscape integration. This
article focuses on the importance of baobab trees in the construction of this urban pole. Our bypothesis is that baobab trees are given
special attention because they are considered a protected species (and are therefore one of Senegal's two symbolic seals), but also because
they provide remarkable ecosystem goods and services. This study is based on inventory data of the baobab trees in the Diamniadio urban
centre and on survey data (quantitative and qualitative). The in situ baobab tree inventory identified 333 trees randomly distributed
within the urban centre. Despite urban growth, baobab trees still maintain their symbolic aspect that is engraved in the collective memory.

\section{Introduction}

La construction de nouvelles villes est un processus à enjeux multiples. Elle suscite plusieurs préoccupations, notamment la question de l'intégration paysagère. Dans ce contexte, la nature devient un élément important à prendre en compte dans la conceptualisation, la construction, l'aménagement et le développement d'une ville. L'arbre en particulier reste au cœur des politiques urbaines, car il participe de façon significative à l'amélioration du cadre de vie de ses résidents et à l'écologie urbaine (Bœuf et Robert, 2017).
La question abordée ici est relative à l'image de l'arbre dans une ville en construction. Ce sujet est en effet peu étudié dans les villes des pays qu'on dit en développement. Cet article s'intéresse plus particulièrement à la place d'un arbre emblématique, le baobab, au cœur du projet d'aménagement d'un pôle urbain, soit celui de Diamniadio, à environ 30 kilomètres de Dakar, au Sénégal.

Ce projet de pôle urbain permet de s'interroger sur la prise en compte du peuplement pluricentenaire de baobabs par les aménageurs, car cette espèce remarquable est à la fois emblématique, symbolique et sacrée; elle bénéficie donc d’un

\footnotetext{
a Enseignant-chercheur, Laboratoire de biogéographie, Université Cheikh Anta Diop, Dakar

b Enseignant-chercheur, Laboratoire de biogéographie, Université Cheikh Anta Diop, Dakar
} 
statut de protection partielle dans le Code forestier du Sénégal. Les objectifs de cet article sont de faire une analyse sur l'état du peuplement et sur le respect ou non du statut de protection du baobab au regard de ses valeurs multiples; de relever les défis de sa préservation dans l'aménagement de la ville de Diamniadio; et de mettre en valeur l'image du baobab dans le cadre de vie de la future ville.

\section{Présentation de la zone d'étude}

La création du pôle urbain de Diamniadio (PUD) est une initiative du gouvernement sénégalais, qui désire impulser une nouvelle dynamique de développement socioéconomique au pays. Elle est fondée sur la mise en place d'une plateforme multifonctionnelle pour l'essentiel des activités productrices de revenus afin d'inciter notamment les entreprises à délocaliser et diversifier leurs activités hors de la capitale (Dakar) et à attirer de nouveaux investisseurs.
Le pôle urbain de Diamniadio a été institué par le Décret $n^{\circ} 2013-1043$ du 25 juillet 2013, qui déclare la zone d'utilité publique. Ce nouveau pôle de développement urbain s'étend sur 1644 hectares. Les principales composantes prévues dans la phase initiale du projet sont :

- un pôle Logements avec 2360 logements d'habitation, dont 1714 villas et 646 appartements;

- un pôle Institutions publiques sur environ 26 hectares;

- un pôle Activités touristiques sur environ 75 hectares;

- un pôle Institutions privées sur environ 2 hectares;

- un pôle Activités tertiaires sur environ 6 hectares;

- divers lieux de culte sur 1 hectare.

Dans le cadre de cette recherche, nous nous sommes limités à la zone initiale du pôle urbain de Diamniadio en cours d'aménagement (voir figure 1).

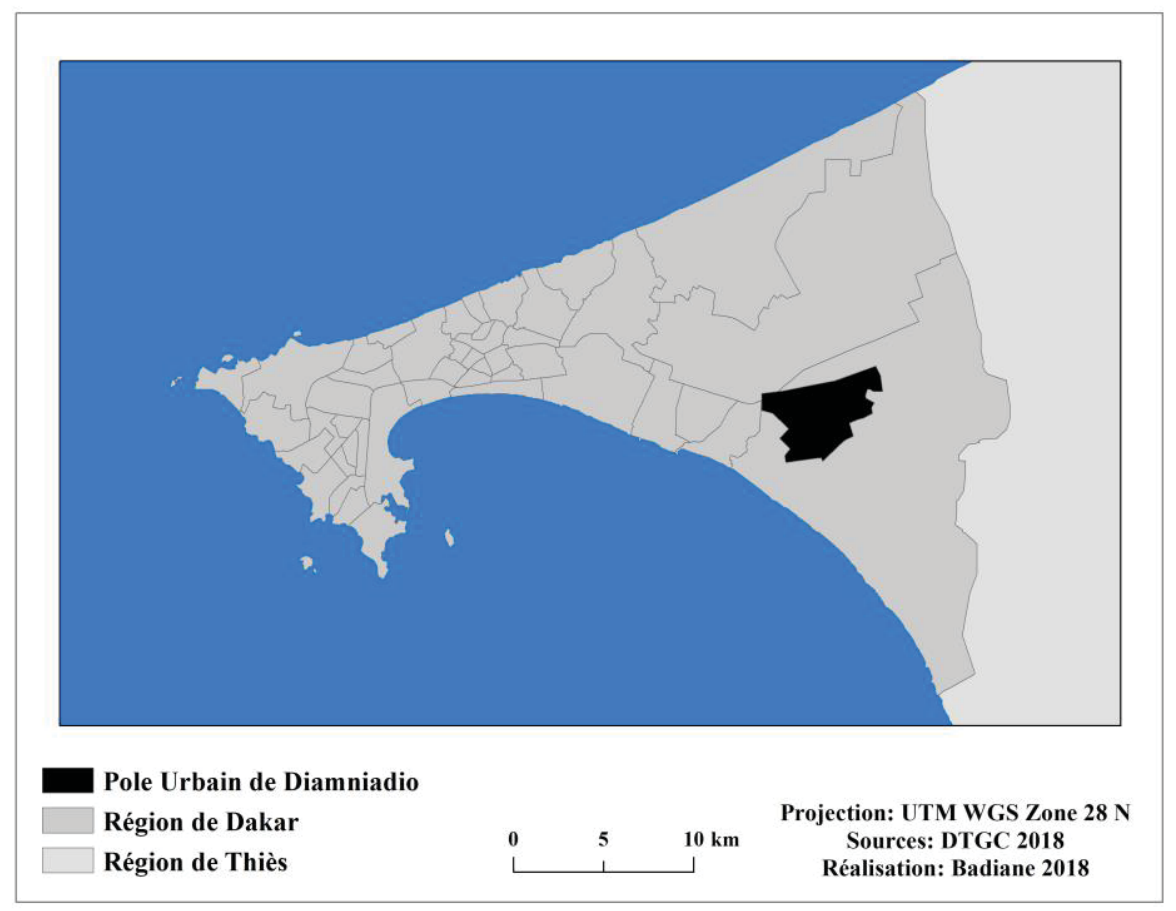

Figure 1 - Situation géographique du PUD Sources : DTGC, 2018

Le pôle urbain de Diamniadio est construit sur le plateau sédimentaire de Rufisque-Bargny, qui appartient au bassin sédimentaire sénégalo-mauritanien du Quaternaire. Cette formation géologique du plateau de Rufisque-Bargny est fortement perturbée et fracturée au sud-ouest/nord-est, élevant les marnes et abaissant les calcaires. Ces discontinuités forment le réseau de drainage principal (Barusseau et collab., 2009). 


\section{De la recherche sur les arbres remarquables à l'intégration paysagère urbaine : démarche}

Une large revue de la littérature a permis de faire l'état des lieux sur la recherche géographique concernant les arbres remarquables, leur intégration paysagère dans les projets d'aménagement urbain, le symbole de l'arbre (passé, présent, avenir) comme véhicule de la mémoire collective ainsi que les théories et méthodes de recherche sur l'arbre en ville.

Ce travail de recherche a nécessité aussi la mobilisation de données primaires de type qualitatif et quantitatif. Une enquête a été réalisée auprès de quelques cibles. Il s'agit d'entrevues semi-dirigées abordant des thèmes tels que l'intérêt patrimonial de l'arbre en ville, ce qu'il représente, les valeurs qu'il véhicule dans l'imaginaire des citadins (ancienneté, identité, paysage), la continuité ou non de sa gestion, etc. Essentiellement, les personnes interrogées sont des aménageurs, des responsables du pôle urbain, des habitants des villages proches du pôle urbain (Diamniadio et Déni Malick Guèye) et des personnes-ressources (p. ex., les agents du service des eaux et forêts et des conseillers municipaux de Diamniadio). Au total, près de 30 personnes au profil divers ont été interrogées.

Les données à caractère phytogéographique (distribution spatiale) et botanique (stade de croissance, appareil végétatif, port, fruits, etc.) du baobab ont été collectées. Cette opération de collecte d'informations a nécessité l'élaboration d'un protocole simplifié d'inventaire floristique en raison de la nature du site, de la physionomie de la végétation et de l'objet de l'étude. L'inventaire, réalisé en décembre 2018, vise à caractériser le peuplement de baobabs. Il s'agit d'estimer le nombre d'individus afin de dresser un état des lieux de la structure du peuplement.

L'étude du peuplement de baobabs de Diamniadio a consisté en un inventaire assez exhaustif de l'espèce. La technique du "tour de champ » a été choisie. Cette méthode de prospection itinérante consiste à inventorier toutes les espèces en parcourant une surface d'observation, définie en fonction de l'hétérogénéité des milieux, dans différentes directions (Noba, 2002).

Revue Organisations \& Territoires • Volume 28 • № $2 \cdot 2019$
Toutefois, dans le cadre de la présente étude, l'attention fut accordée au baobab seulement. Le comptage phytosociologique systématique a été privilégié, compte tenu de la physionomie du paysage arboré assez éparse et de la forte anthropisation du site. Cette approche permet d'obtenir des données aussi complètes que possible. Or, il y a lieu de signaler que certaines zones inaccessibles ont été exclues (essentiellement des domaines privés et des sites de chantier). Les paramètres dendrométriques mesurés sont notamment la hauteur, la circonférence, le stade de développement et l'état de santé. La localisation des arbres a été faite à l'aide d'un système de localisation GPS; la mesure de la circonférence grâce à un mètre à ruban; l'estimation de la hauteur par une perche; et le comptage phytosociologique avec des fiches de relevé. L'identification des espèces a été réalisée à partir des ouvrages sur la flore du Sénégal de Berhaut (1967/1971/1991).

Les données d'inventaire floristique ont été dépouillées, puis traitées avec le logiciel tableur Excel. L'exploitation de ces données permet d'analyser les paramètres indiqués plus haut puisque l'objet ici est de connaitre la composition floristique et de discuter l'intérêt de la conservation de l'arbre emblématique du Sénégal.

\section{Patrimoine arboré dans une ville en construction : le pôle urbain de Diamniadio}

Le peuplement de baobabs sur l'étendue du pôle urbain de Diamniadio est constitué de 333 individus aux caractéristiques très variables. Ces baobabs ont une répartition très éparse sur le territoire et le cortège floristique semble être très affecté du fait que le site est en cours d'artificialisation, c'est-à-dire en perte des qualités qui sont celles du milieu naturel.

\subsection{Caractéristiques du peuplement de baobabs à Diamniadio}

Dans la zone de Diamniadio, le maigre peuplement de baobabs (voir figure 2) est identifié comme le résidu d'un parc arboré jadis important. Ce paysage résulte d'une forme de patrimonialisation de la nature en raison de pratiques non agraires, mais aussi en raison d'autres valeurs qui vont au-delà de celles intrinsèques à l'arbre. Le peuplement de baobabs garde toujours une apparence remarquable, même dans des conditions de dégradation du 
milieu. L'image d'un paysage dégradé garde paradoxalement un certain attrait par la présence de cet arbre tropical dont certains caractères botaniques méritent d'être rappelés ici. Fortin, Lo et Maynart (1997) donnent la description suivante :

Arbre haut de 10 à $15 \mathrm{~m}$ de haut, à tronc énorme atteignant plusieurs mètres de diamètre; écorce lisse et grisâtre. Les branches sont très robustes, peu nombreuses et étalées. Les feuilles sont composées digitées, alternes, ayant un nombre variable (6 à 8) de folioles obovales longues de 10 à $20 \mathrm{~cm}$ ou davantage. $[\ldots]$ Les fruits subsphériques ou ovoïdes de 15 à $35 \mathrm{~cm}$ de long sont ligneux, verdâtres et veloutés (Fortin, Lo et Maynart, 1997, p. 54).

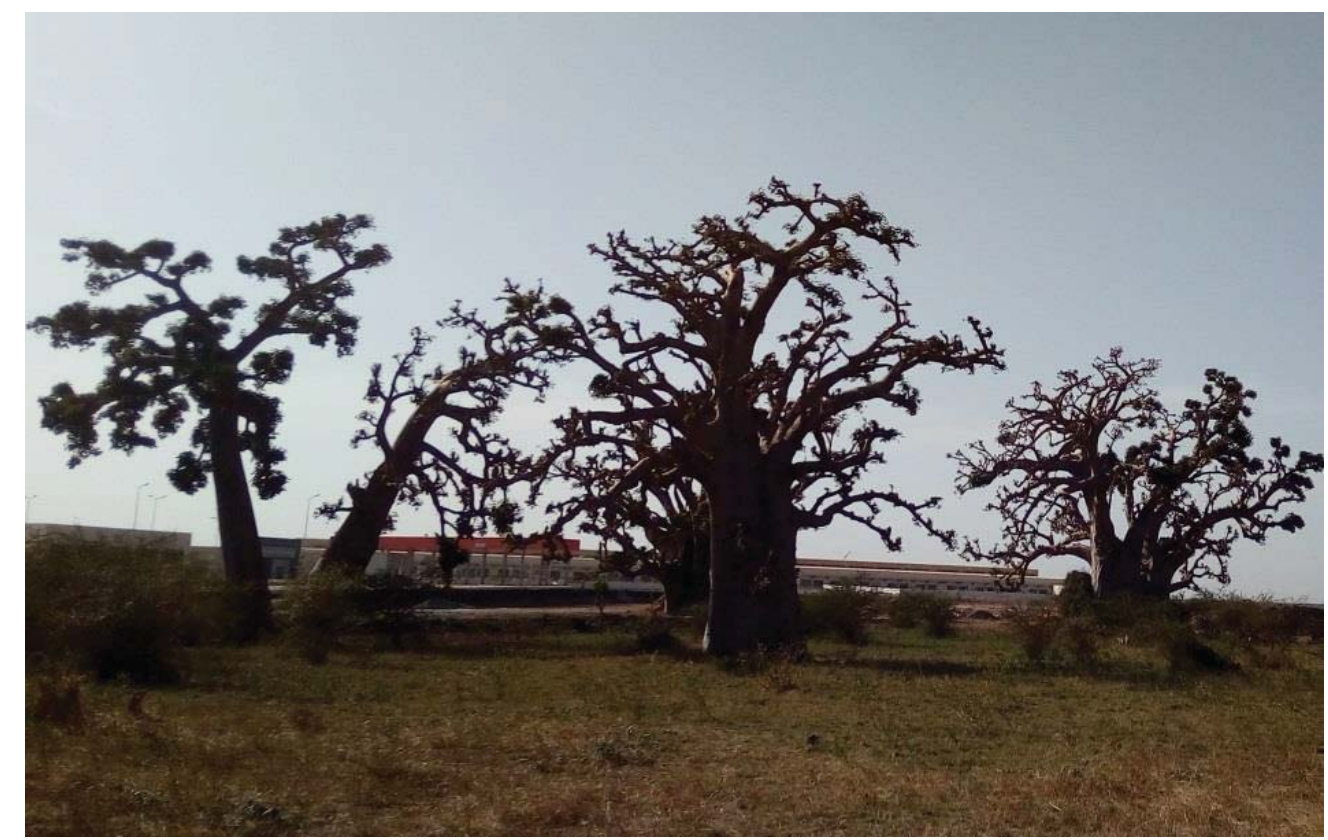

Figure 2 - Regroupement de baobabs, près de la Gare des gros porteurs de Diamniadio Source Badiane et Mbaye, 2018)

Au Sénégal, le baobab pousse sur des terres légères et sablonneuses ou calcaires. Les peuplements les plus importants sont répertoriés dans les environs de Dakar, de Thiès et de Kédougou, en général près des villages. Nous avons rencontré un nombre d'individus plus important dans la zone 2, située dans le quart nord-est du pôle urbain de Diamniadio. Une concentration particulière est observée dans le village de Déni Malick Guèye, dans la commune de Diamniadio. Les autres secteurs où l'on retrouve plus d'individus sont les zones proches de la voie 70 dans le sens nord-sud, notamment à proximité du Marché d'intérêt national, de la Gare des gros porteurs et de la Gare emblématique du Transport express régional (voir figure 3 ). 


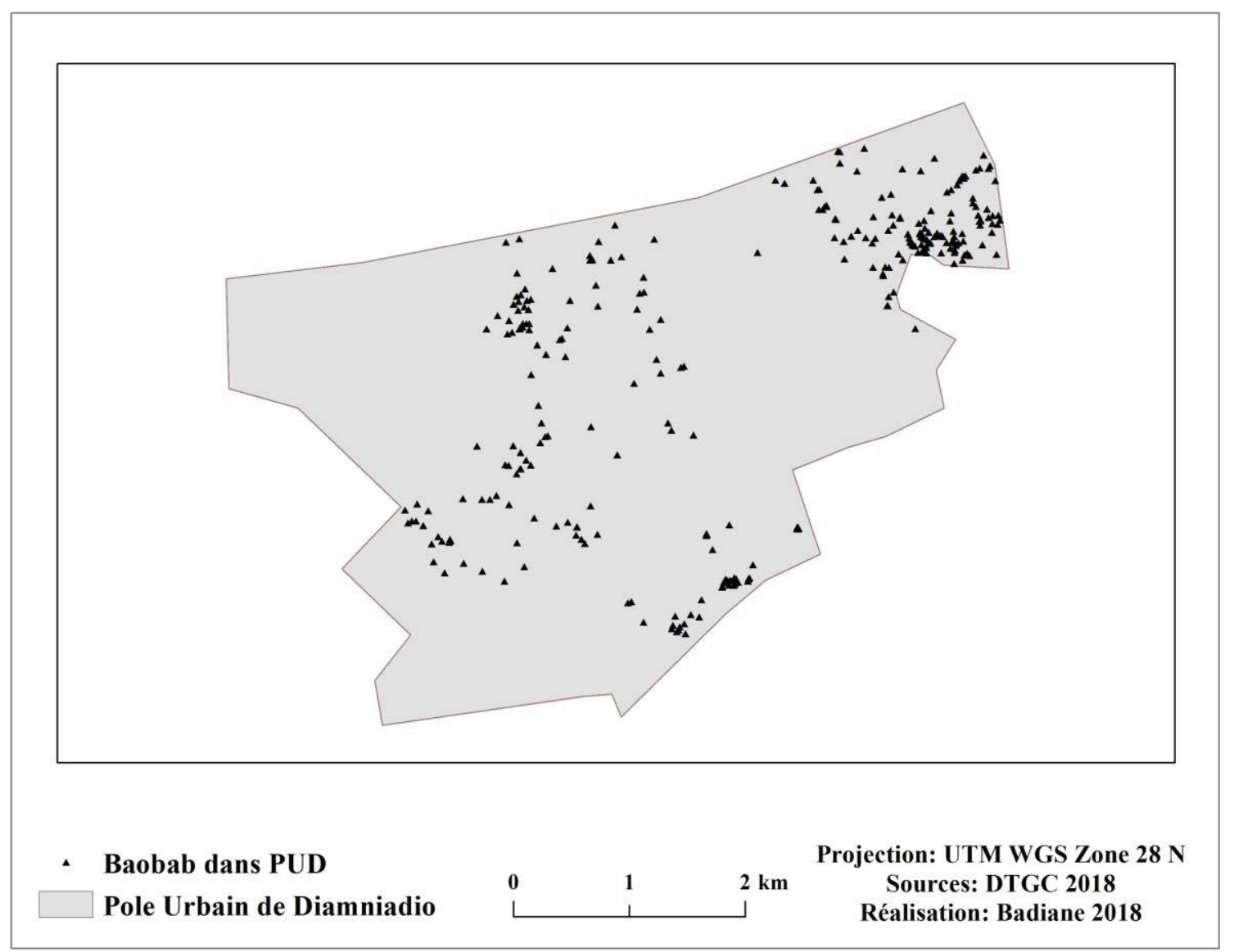

Figure 3 - Distribution spatiale du peuplement de baobabs dans le PUD Sources : DTGC, 2018

L'examen de quelques paramètres dendrométriques des individus inventoriés dans le pôle urbain de Diamniadio indique des éléments particuliers d'appréhension du peuplement de baobabs. Cette analyse se concentre sur le stade de développement, sur la structure verticale, sur la circonférence et sur l'état de santé des individus.

Il faut souligner d'abord que les sujets rencontrés sont répartis en trois classes correspondant aux étapes de croissance des arbres (Sabatier, Caraglio et Drénou, 2014). L'interprétation de la figure 4A révèle que les individus matures ont la proportion la plus importante, soit $54 \%$ des individus répertoriés. Cette catégorie est suivie de celle des individus vieillissants, puis des sujets jeunes. La figure $4 \mathrm{~B}$ rend compte de la structure verticale du peuplement de baobabs. Les individus ont été classés selon la hauteur, laquelle varie de moins de $5 \mathrm{~m}$ à plus de $20 \mathrm{~m}$. Il apparait que la classe des $5-10 \mathrm{~m}$ domine, soit $28 \%$ des individus. Elle est suivie de la classe des 10-15 m, des 15-20 m et des moins de $5 \mathrm{~m}$. La classe de hauteur de plus de $20 \mathrm{~m}$ est faiblement représentée. L'analyse de la figure 4C montre la répartition des classes de circonférence des individus. La catégorie des individus de moins de $5 \mathrm{~m}$ domine. Elle est suivie par la classe des individus des 5-10 m, ensuite par celle des 10-15 m. Les individus de plus $15 \mathrm{~m}$ arrivent en dernière position. L'état de production des individus est représenté dans la figure $4 \mathrm{D}$, où l'on remarque que $97 \%$ des individus sont non productifs, contre $3 \%$ d'individus productifs. 


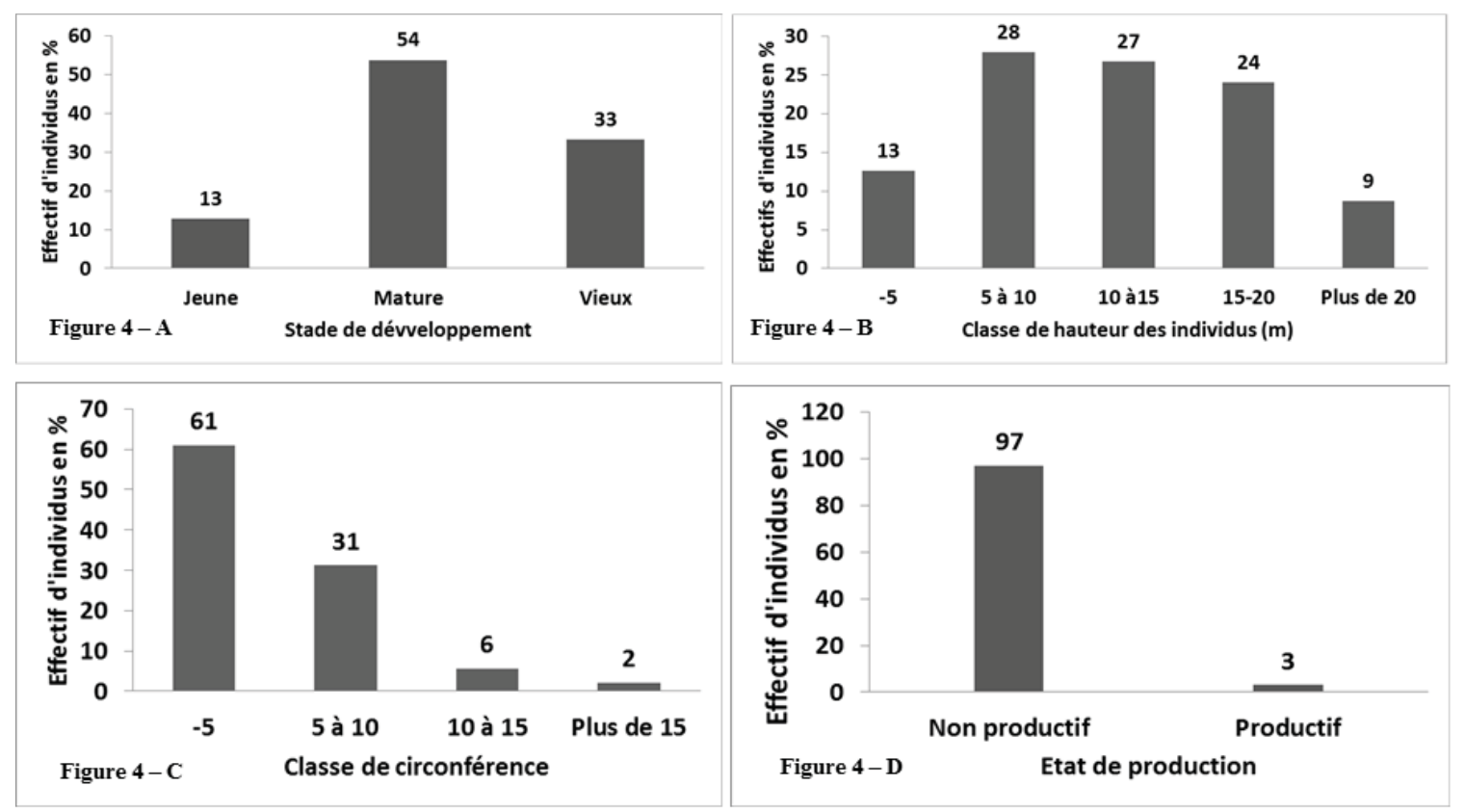

Figure 4-Synthèse de quelques paramètres dendométriques du peuplement de baobabs du PUD Source : Badiane et Mbaye, 2018

Le peuplement est constitué essentiellement d'individus adultes, ce qui témoigne de l'équilibre relatif du parc à baobabs de Diamniadio. La hauteur et la circonférence sont des indicateurs assez parlants de l'état de maturité des individus. Cependant, la plupart des individus ne produisent pas de fruits au moment de l'inventaire. Le peuplement présente des signes de sénescence précoce, probablement due au stress. Les arbres subissent une pression énorme en raison de leurs usages multiples. Les feuilles sont cueillies pour la fabrication d'une poudre alimentaire ou pour servir de fourrage au bétail, ce qui ne favorise pas l'activité de photosynthèse essentielle à la vie de l'arbre. D'autres parties de l'arbre sont également très prisées. L'écorce est utilisée pour confectionner des cordages traditionnels et les racines sont employées dans la pharmacopée traditionnelle.

\subsection{Effet des aménagements du pôle urbain de Diamniadio sur le peuplement de baobabs}

Pour apprécier l'impact de l'aménagement du pôle urbain sur le peuplement de baobabs, il a été judicieux de partir des données officielles issues des documents d'étude d'impact environnemental de certains projets. Ces sources ont été complétées par les observations directes lors de l'activité d'inventaire des baobabs sur le site. Quelques témoignages ont été recueillis lors de l'entrevue auprès des aménageurs.

La mise en place des infrastructures a nécessité parfois l'abattage de baobabs. Par exemple, dans le rapport provisoire d'étude d'impact environnemental et social du projet de construction du Marché d'intérêt national (MIN) et de la Gare des gros porteurs (GGP), il est indiqué que « les travaux de construction du MIN et de la GGP vont nécessiter l'abattage de deux baobabs » (Souley, 2015, p. 77).

Nous avons effectivement constaté des coupes de baobabs parfois de gros calibre sur les terrains de terrassement (voir figure 5). La perte du peuplement de baobabs induite par les projets d'aménagement urbain est donc une réalité. Dans un domaine privé situé à côté de la Gare emblématique du Train express régional, 11 baobabs ont été dénombrés. Le propriétaire envisage d'abattre 9 individus pour l'installation d'un hangar. 

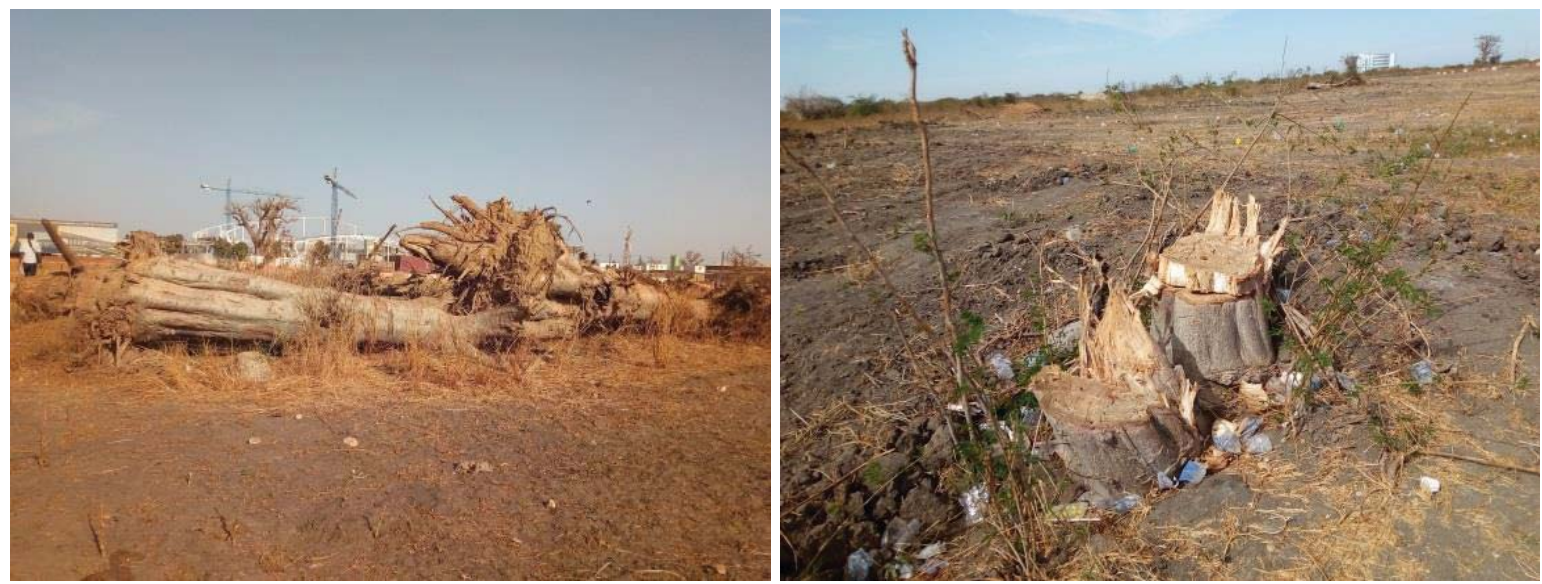

Figure 5 - Baobabs abattus dans le PUD

Source : Badiane et Mbaye, 2018

Même quand la coupe est autorisée par les services compétents, il est parfois regrettable de voir disparaitre progressivement cette espèce emblématique dans un contexte d'urbanisation. La situation du peuplement de baobabs à Diamniadio pourrait être plus inquiétante en raison des contraintes biophysiques du milieu.
L'état de santé des baobabs répertoriés le prouve déjà, car, sur les 333 individus de cette étude, 29\% présentent des signes de maladie, $2 \%$ sont constitués de souches (avec ou sans rejets) et $1 \%$ sont des individus morts (voir figure 6). Au total, le peuplement comporte $32 \%$ d'individus malsains.

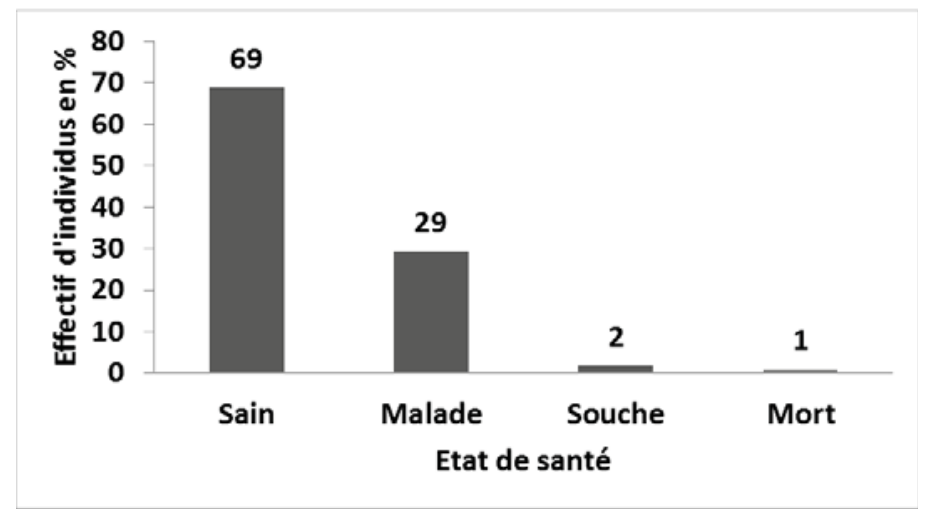

Figure 6 - État de santé du peuplement de baobabs Source : Badiane et Mbaye, 2018

La figure 7 montre la répartition spatiale des individus selon l'état de santé. Il apparait que les souches se retrouvent généralement dans les endroits en cours d'aménagement. Les individus sains et malades présentent presque la même configuration de distribution spatiale. 


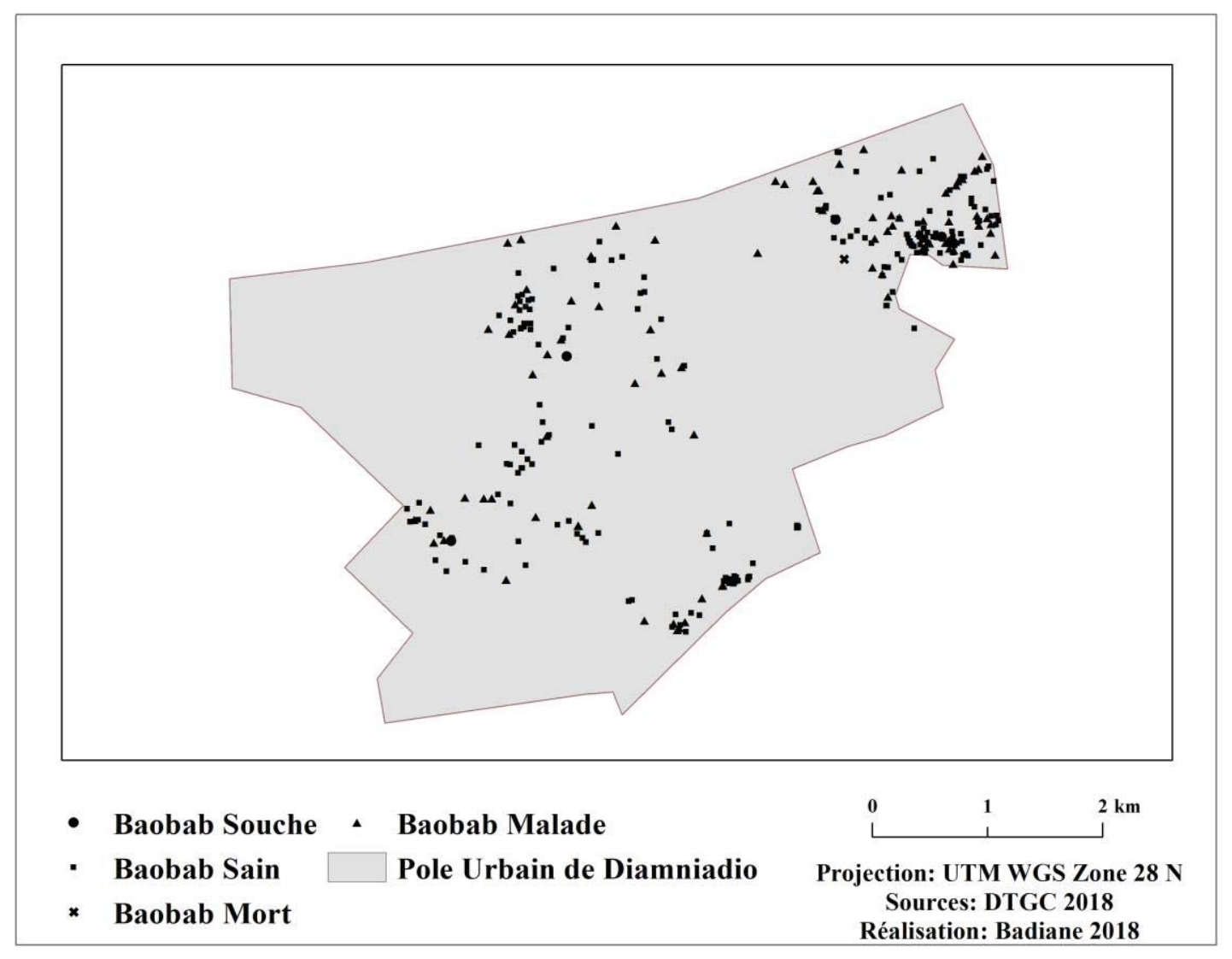

Figure 7 - Distribution spatiale des individus selon l'état de santé Sources : DTGC, 2018

Le fait que $32 \%$ des individus soient malsains ou malades indique que la situation n'est pas favorable au maintien des baobabs dans la future ville de Diamniadio. Il faudra donc de nombreux efforts des acteurs (État, collectivité, citadins) pour garantir la présence de cet arbre dans une ville en construction, où le « tout en béton » sera fatalement l'architecture qui s'imposera dans cet espace urbain. Par contre, il peut y avoir des paradoxes de survie des baobabs, comme on l'observe à Dakar, avec des milliers, voire des dizaines de milliers d'individus au cour de la ville (Ndiaye, 2015). Dans certains quartiers centraux de Dakar, les baobabs ont résisté à l'urbanisation et font désormais partie du décor.

\section{Le baobab en tant qu'emblème du Sénégal et sa représentation à Diamniadio}

Le baobab fait partie des arbres remarquables dans le paysage forestier de Diamniadio. Il en ressort qu'il occupe une place majeure dans les représentations sociales de la nature chez les populations de cette nouvelle ville. On reconnait de ce fait les valeurs multiples du baobab (symbolique, économique, sociale, historique, mythique), malgré les mutations socioculturelles intervenues au cours du temps dans la zone métropolitaine de Dakar.

\subsection{Représentation collective autour du baobab}

Il n'est pas rare de rencontrer le baobab dans les zones d'habitation au Sénégal. L'histoire de cette bombacée réside en ce qu'elle fait partie des éléments constituant l'emblème du pays. Établi comme l'un des deux sceaux du Sénégal, le baobab figure dans la deuxième moitié de l'écusson (voir figure 8). Il est donc un symbole national. Ce texte extrait du site officiel de la Présidence de la République du Sénégal ${ }^{1}$ indique que :

Le baobab est le sceau commun qui estampille les actes de toute l'administration publique de la République du Sénégal. Le baobab est d'abord un arbre typique de la flore sénégalaise. Un groupe 
de baobabs centenaires marque, souvent, la place d'un ancien village. C'est ensuite un arbre dont les paysans tirent de multiples ressources. Le fruit, le pain de singe, sert à fabriquer la sauce de bouillie. Les feuilles pilées entrent dans la composition du couscous. Le tronc fournit des cordes solides.

La Police nationale a adopté comme symbole particulier le baobab, que l'on retrouve sur ses signes distinctifs (insigne, épaulettes, logos, etc.).
Sur le sceau du baobab est inscrit : République du Sénégal-Au nom du peuple sénégalais.

Les sceaux, timbres et cachets des grands corps de l'État, des ministères, des cours et tribunaux, des notaires, de toutes les administrations et autorités publiques porteront pour type le baobab tel qu'il est figuré sur le sceau de l'État et pour légende "République du Sénégal » ainsi que le timbre de l'administration ou de l'autorité publique pour laquelle ils seront employés (article 2 de l'ordonnance n⿳60-26 du 10 octobre 1960).

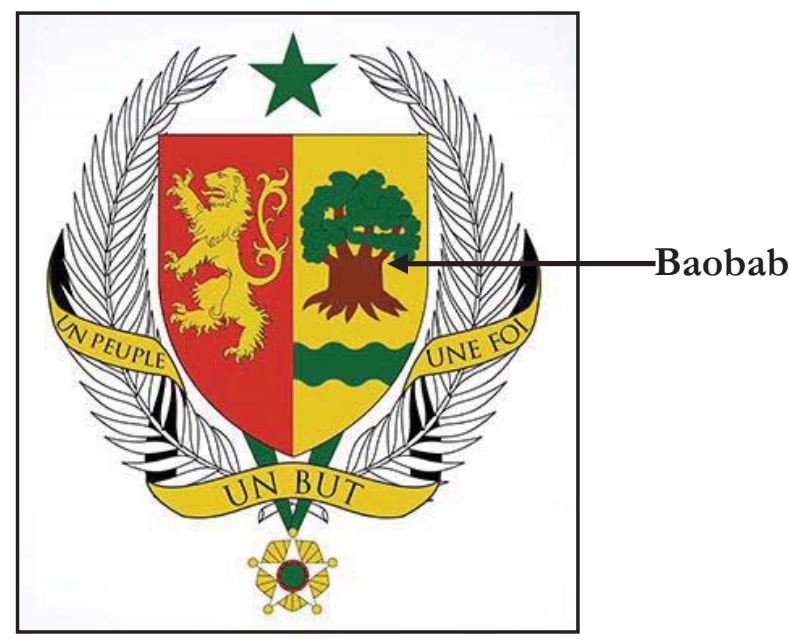

Figure 8- Le baobab, un des deux sceaux du Sénégal Source : www.presidence.sn/presidence/le-sceau

Ces considérations fondent le lien profond entre le baobab et le peuple sénégalais. Cet arbre inspire le respect partout au Sénégal, en milieu rural comme en ville, dans les espaces les plus insignifiants comme dans ceux les plus tentaculaires. Qu'il soit isolé ou en groupe, le baobab est bien présent dans la mémoire collective des Sénégalais. Son institutionnalisation comme symbole national renforce l'identité commune. Cette apparence est encore plus marquée en ville, où les maigres peuplements de baobabs peuvent être jalousement gardés pour diverses raisons (Ndiaye, 2015). En effet, $90 \%$ des personnes interrogées au cours de cette recherche sur le site du pôle urbain de Diamniadio reconnaissent l'intérêt patrimonial du baobab. Un interlocuteur dit à ce propos : "Quand je vois un baobab, ça me renvoie à mon enfance. J'ai grimpé sur tous ces baobabs que tu vois quand j'étais enfant. » Cela revient à dire qu'il y a une résistance mémorielle pour cet interlocuteur, qui lui fait regretter le mauvais traitement fait à ces arbres en ces termes :
Les baobabs se raréfient de plus en plus; avant, on les rencontrait partout, mais maintenant on en voit difficilement. Il y avait des individus de cette espèce qui sont attribués aux griots, certains d'entre eux se trouvaient de l'autre côté de l'autoroute à péage, mais il n'y en a plus, sinon j'allais vous y amener.

\subsection{Valeur perçue et valeur réelle du baobab : entre représentation et réalité à Diamniadio}

Le baobab est connu comme une espèce végétale partiellement protégée par le Code forestier du Sénégal de 1998, révisé en 2019. Ce fait est bien compris par $68 \%$ des personnes interrogées. La question des valeurs de cette espèce reste assez présente dans le discours des interlocuteurs. Au-delà de cette perception symbolique (sceau du Sénégal), le baobab affiche des usages multiples qui renforcent son lien avec certains territoires sénégalais et leurs habitants. Il a 
une forte représentation sociale. Ainsi, $95 \%$ des personnes interrogées reconnaissent les valeurs (alimentaire, médicinale) de cet arbre. À la question de savoir si l'arbre exprime une image particulière (représenta-

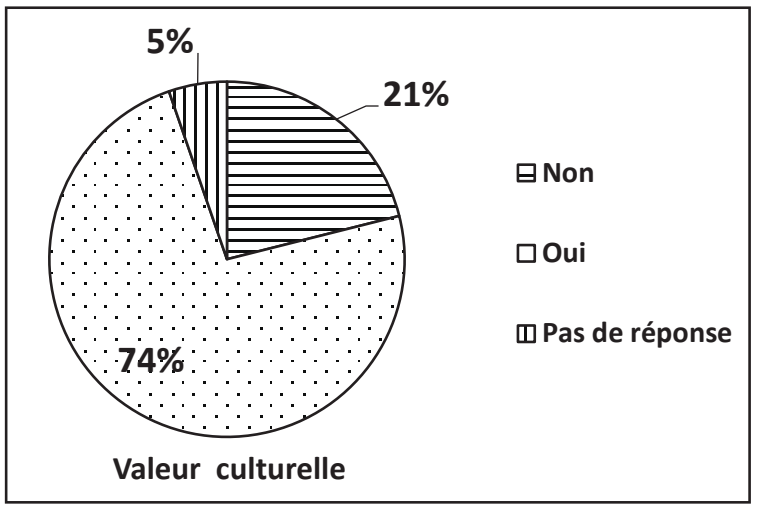

tion sociale, culturelle, mystique) ou une tradition religieuse, $74 \%$ ont répondu positivement (voir figure 9). Le même résultat est obtenu concernant l'appréciation de la valeur économique du baobab.

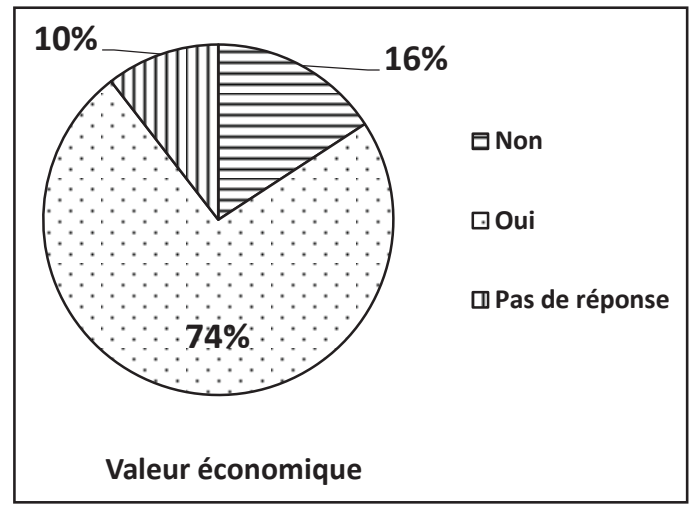

Figure 9-Valeurs du baobab

Source : Donnée d'enquête (Badiane et Mbaye, 2018)

L'évaluation globale de la valeur d'usage de l'arbre (alimentaire, thérapeutique, artisanal, etc.) a obtenu une note de $5 / 5$ d'après $63 \%$ des personnes interrogées. Un des interlocuteurs dit que «tout le monde connait son importance; toutes ses composantes ont de la valeur ».

Par ailleurs, l'enquête ethnobotanique de notre recherche a permis de relever les nombreux services rendus par le baobab aux populations. Nous avons pu recueillir cette affirmation d'un habitant du village de Déni Malick Guèye :

Les feuilles du baobab donnent de la poudre de baobab et, quand on était enfant, quand on avait mal aux yeux, nos mamans prenaient la poudre ou les feuilles pour nous nettoyer le visage. Je sais aussi que la sève soigne les maux de dents. Le pain de singe est utilisé pour soigner les brûlures, mais aussi pour l'alimentation. Je connais d'autres vertus de cet arbre, mais je préfère les garder pour des raisons mystiques.

Bien plus qu'un simple arbre, le baobab fait partie intégrante de l'identité des communautés locales. Il est associé aux croyances locales. Il n'est pas rare d'entendre des témoignages selon lesquels certains baobabs sont sacrés. Nous avons relevé au moins trois témoignages dans ce sens dans le seul village de Déni Malick Guèye. L'intégration de ce village dans le pôle urbain de Diamniadio ne changera certainement pas le statut sacré de ces baobabs.

\section{Le baobab au cœur de l'imaginaire urbain}

L'intégration paysagère est en effet prise en compte dans la création de la nouvelle ville de Diamniadio. On peut facilement identifier, dans le plan d'aménagement de ce pôle urbain, les éléments paysagers comme la «coulée verte» ou encore la «coulée bleue » pour parler de la valorisation des espaces verts et des plans d'eau.

Le baobab figure en bonne place dans la politique urbaine en matière d'intégration paysagère, du moins selon les documents définissant les lignes directrices de l'aménagement du pôle urbain, en l'occurrence le Plan directeur d'urbanisme de Dakar et ses environs : horizon 2035. Il était donc légitime de s'interroger sur les faits présents et sur la conception du futur urbain avec une dimension phytogéographique du baobab.

\subsection{De la valeur intrinsèque d'un arbre emblématique à son intégration dans l'imaginaire urbain}

En nous intéressant à la valeur intrinsèque du baobab perçue par les acteurs locaux, nous leur avons posé la question suivante: Quels sont les éléments remarquables du baobab dans son apparence? Les réponses obtenues restent très variées (voir figure 10). Il ressort que les feuilles de l'arbre associées à la fleur sont bien appréciées, ensuite les fruits, puis le port et enfin l'écorce. Il faut comprendre que le baobab est connu 
dans cette ancienne zone rurale pour son rôle important dans l'apport alimentaire par ses feuilles et ses fruits (Baumer, 1995). Malheureusement, très peu de baobabs gardent actuellement leurs feuilles en raison de l'émondage fait par les populations locales. L'écorce est aussi généralement prélevée pour la fabrication de cordes.

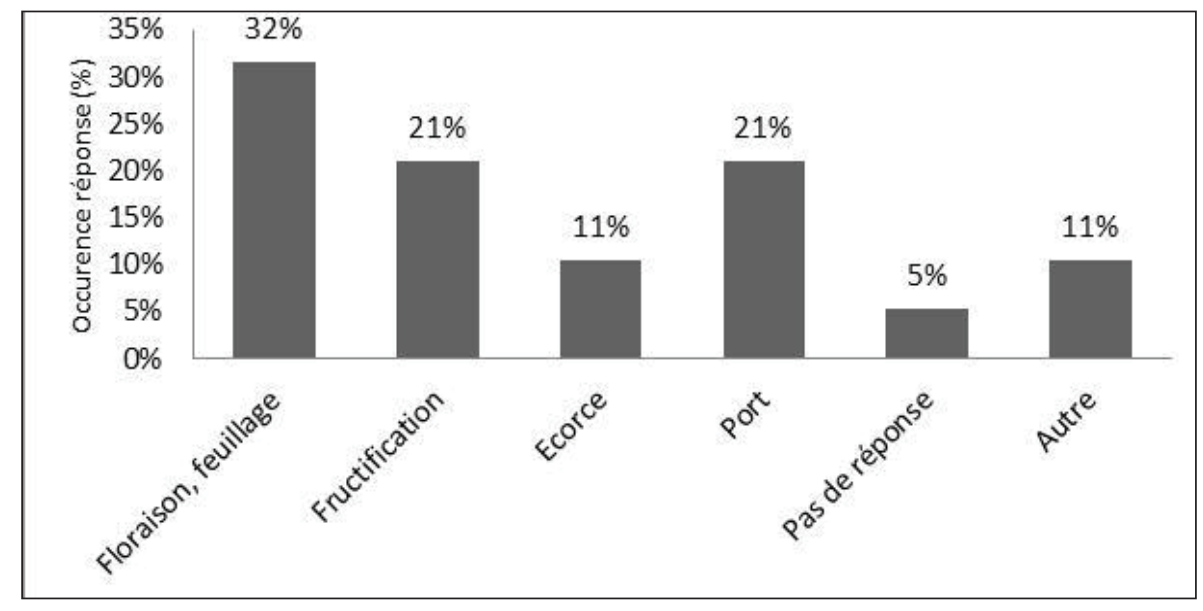

Figure 10 - Valeurs intrinsèques du baobab

Source : Donnée d'enquête (Badiane et Mbaye, 2018)

Le port de l'arbre est souvent remarquable par sa dimension, gigantesque du point de vue de l'allure verticale et de la grosseur du tronc. Par exemple, le plus gros calibre rencontré mesure $210 \mathrm{~cm}$ de circonférence à hauteur de poitrine. Cette apparence justifie parfois les mesures de conservation de l'espèce dans des territoires comme Diamniadio. Dans un communiqué ${ }^{2}$ du Conseil des ministres du 11 juin 2018, on peut relever l'importance accordée à la préservation du baobab à Diamniadio par le président de la République du Sénégal :

Appréciant, par ailleurs, l'importance de la préservation de l'écologie de Diamniadio et du lac Rose, le président de la République souligne que des promoteurs privés et services de l'État procèdent au déracinement systématique des baobabs pendant l'exécution des projets à Diamniadio et sur le site du lac Rose.

Ainsi, le président de la République demande au premier ministre de faire prendre les dispositions nécessaires en vue de l'interdiction du déracinement des baobabs sur les sites de Diamniadio et du lac Rose, notamment en le notifiant, dans les meilleurs délais, aux services de l'État concernés et aux promoteurs privés.

Le chef de l'État indique enfin le prix qu'il attache à la préservation de l'écosystème du lac Rose et notamment celui de Diamniadio, qui doit répondre à sa vocation de ville verte où un projet d'aménagement paysager est d'ailleurs prévu à ce titre.

Bref, le cas du pôle urbain de Diamniadio reste une véritable expérience. Tout porte à croire que, même dans le contexte d'urbanisation tous azimuts, le baobab sera conservé. Sa préservation reste une préoccupation exprimée au plus haut palier du gouvernement sénégalais, même s'il existe encore des interrogations sur les moyens à mettre en œuvre pour relever ce défi.

L'apparence d'un baobab suffit parfois à lui accorder une image d'arbre mythique, c'est-à-dire autour duquel est construite une croyance animiste chez certaines communautés du Sénégal. Comme le raconte un habitant de Déni Malick Guèye, «tous les baobabs abritent des génies ». Cette perception très populaire du baobab lui vaut même le statut d'arbre sacré. De ce fait, certains baobabs font office de lieu de culte où les populations organisent des cérémonies religieuses, des sacrifices, des libations, etc.

Nous avons identifié quelques-uns de ces baobabs considérés comme étant sacrés dans l'espace du pôle urbain de Diamniadio. D'après les responsables de la Délégation générale à la promotion des pôles urbains, il y a une réelle volonté politique 
pour que les baobabs soient conservés dans la nouvelle ville de Diamniadio. Ils marquent le paysage urbain et sont considérés comme des éléments intrinsèques du patrimoine présent et futur de la ville.

\subsection{La transplantation}

de baobabs : entre transformation sociospatiale programmée et maintien d'un patrimoine naturel et historique en milieu urbain

La présence de baobabs peut faire l'objet d'une réinterprétation sociale. Comme l'expriment Berdoulay, Castro et Da Costa Gomes (2001) :

Il y a donc ici une forme d'interdépendance sociospatiale de contenu politique et territorial objectivée dans l'imaginaire social. Il s'agit alors de proposer que tout imaginaire social, de même qu'il possède une forte composante
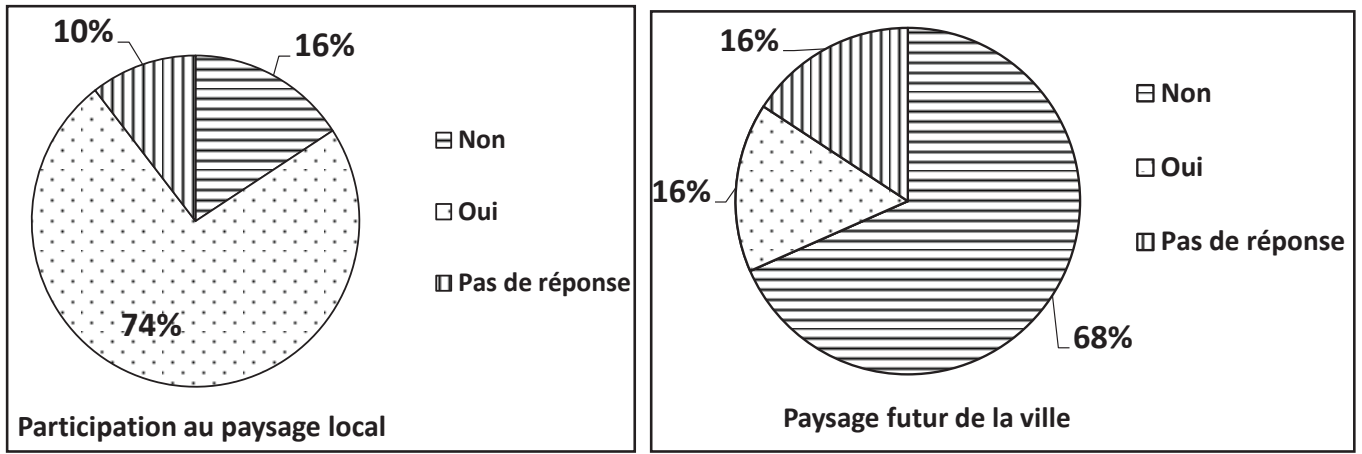

Figure 11 - Valeurs paysagères du baobab

Source : Donnée d'enquête (Badiane et Mbaye, 2018)

En tant qu'élément du patrimoine naturel et historique, le baobab est associé en partie au processus de transformation spatiale de Diamniadio. Quelques faits pourraient illustrer la forte appropriation de l'image du baobab dans le projet de ville. Il s'agit notamment de la replantation d'un baobab à l'esplanade de la Plateforme industrielle internationale de Diamniadio, inaugurée en 2015 et qui devrait abriter des industries manufacturières non polluantes. L'arbre replanté, bien en évidence, marque l'entrée principale du domaine industriel.

Cette action a inspiré certains aménageurs, qui envisagent d'utiliser la même idée dans d'autres secteurs du pôle urbain. Il faut signaler que cette politique, possède également une autre composante spatiale par l'intermédiaire du pouvoir symbolique attribué aux objets géographiques, naturels ou construits, qui sont en relation directe avec une existence humaine (Berdoulay et collab., 2001, p. 422).

L'image du baobab semble marquer son empreinte, comme le démontre le symbole du sceau de la nation sénégalaise. D'ailleurs, la Gare emblématique du Train express régional de Diamniadio doit son appellation de "gare emblématique » à la présence de cinq baobabs dans son périmètre. C'est une sorte de stabilisation de la mémoire autour de ces baobabs qui se tiennent désormais de façon majestueuse dans l'espace de la gare. De ce point de vue, l'arbre participe à définir le paysage local, selon $74 \%$ des personnes interrogées (voir figure 11). C'est pourquoi à la question de savoir si les arbres risquent de nuire à l'appropriation du paysage urbain dans le futur, $68 \%$ des personnes interrogées ont répondu « non ». technique a été réalisée dans d'autres villes, comme le soulignent Bœuf et Robert (2017).

Au-delà de l'imaginaire et de la symbolique, le baobab pourrait, sur le plan concret, être davantage apprivoisé par les futurs citadins. D’ailleurs, la forte connotation culturelle impose le respect de cet arbre, et le développement urbain ne créera probablement pas une rupture avec ce mythe, car «les récits traditionnels continuent à être considérés comme pleins de sens » (Claval, 2001, p. 353). Cette perspective semble entretenir une idée forte de la persistance de la mémoire collective autour du baobab dans le contexte du pôle urbain de Diamniadio. 


\section{Conclusion}

Cette recherche pose la question du maintien d'un élément du patrimoine naturel, c'est-à-dire le baobab, dans un contexte de construction d'une nouvelle ville. Il s'agit bien d'une production programmée de territoire urbain qui essaie de prendre en compte les aspects de mémoire collective à travers le baobab, un arbre emblématique.

Les enseignements principaux qu'on peut tirer de cette étude portent sur la réminiscence des symboles institutionnalisés ainsi que sur la mise en cohérence du symbolique et du réel en rapport avec la production de l'espace. Les résultats montrent l'image d'un objet naturel considéré comme emblématique et de plus en plus approprié dans le projet d'aménagement urbain. Le baobab devient sans doute un élément du paysage urbain, car il mérite une attention particulière. Cela incite à pousser la recherche sur les pratiques urbaines et sur l'image de l'arbre, surtout lorsqu'il est une espèce protégée en raison de sa valeur patrimoniale et de la réduction du peuplement, notamment dans les zones urbaines.

\section{NOTES}

1 Présidence de la République du Sénégal (...). Le sceau. Repéré à www.presidence.sn/presidence/le-sceau

2 Secrétaire général du Gouvernement. Sénégal (2018). Communiqué du Conseil des ministres du lundi 11 juin 2018. Repéré à www.mesr.gouv.sn/ communique-du-conseil-des-ministres-du-lundi-11-juin-2018

\section{RÉFÉRENCES}

Barusseau, J.-P., Castaigne, P., Duvail, C., Noël, B. J., Nehlig, P., Roger J., Serrano O., Banton, O., Comte, J.-C. et Travi Y. (2009). Notice explicative de la cartographie multi-couches à 1/50 000 et 1/20 000 de la zone d'activité du Cap-Vert. Dakar, Sénégal : Ministère des Mines, de l'Industrie et des PME, Direction des mines et de la géologie. Repéré à www.researchgate.net/ profile/Pierre_Nehlig/publication/273861722_Notice_explicative_de_la_cartographie_ multi-couches_a_150_000_et_120_000_de_la_zone_d\%27activite_du_Cap-Vert_

Senegal/links/550ee4750cf21287416acb9d/Notice-explicative-de-la-cartographie-multi-couches-a-1-50-000-et-1-20-000de-la-zone-dactivite-du-Cap-Vert-Senegal.pdf

Baumer, M. (1995). Arbres, arbustes et arbrisseaux nourriciers en Afrique occidentale. Dakar, Sénégal : Enda-Éditions.

Berdoulay, V., Castro, I. et Da Costa Gomes, P. C. (2001). L'espace public entre mythe, imaginaire et culture. Cabiers de géographie du Québec, 45(126), 413-428. doi: 10.7202/023001ar

Berhaut, J. (1967). Flore du Sénégal. 2e éd. Dakar, Sénégal : Clairafrique.

Berhaut, J. (1971/1991). Flore illustrée du Sénégal. Dakar, Sénégal : Gouvernement du Sénégal, MDR/DEF.

Bœuf, F. et Robert, F. (2017). L'arbre au cœur du paysage urbain : symbole ou action militante? Projets de paysage : Revne scientifique sur la conception et l'aménagement de l'espace, 16. Repéré à www.projetsdepaysage.fr/fr/1_arbre_au_coeur_du_ paysage_urbain_symbole_ou_action_militante_

Claval, P. (2001). Mythe et connaissance scientifique dans l'histoire de la pensée géographique. Cabiers de géographie du Québec, 45(126), 349-368. doi: 10.7202/022998ar

Fortin, D., Lo, M. et Maynart, G. (1997). Plantes médicinales du Sahel. Dakar, Sénégal : Enda-Éditions Tiers-monde.

Noba, K. (2002). La flore adventice dans le sud du bassin arachidier (Sénégal) : structure, dynamique et impact sur la production du mil et de l'arachide (Thèse de doctorat inédite). Université Cheikh Anta Diop, Dakar.

Ndiaye, P. (2015). Le suivi d'un grand arbre... Dans quels buts? Revue Espaces et sociétés en mutations. Département de géographie, Université Cheikh Anta Diop de Dakar, 31-45.

Sabatier, S., Caraglio, Y. et Drenou, C. (2014). L'architecture des arbres au service des forestiers. Innovations agronomiques, 41 , 119-128. Repéré à www6.inra.fr/ciag/content/download/5511/42157/file/Vol41-10-Sabatier.pdf

Souley, M. L. (2015). Étude d'impact environnemental et social : projet de construction d'un marché d'intérêt national et d'une gare de gros porteurs. Rapport provisoire. 